INTERNATIONAL JOURNAL OF MULTIDISCIPLINARY RESEARCH AND ANALYSis

ISSN(print): 2643-9840, ISSN(online): 2643-9875

Volume 04 Issue 09 September 2021

DOI: 10.47191/ijmra/v4-i9-10, Impact Factor: 6.072

Page No.- 1261-1267

\title{
Perceived Health Concerns During COVID 19 Crisis Amongst Jamaicans
}

\author{
Sukanya Prasad MBBS, $\mathrm{MPH}^{1,2,3}$, Liliane Smatt $\mathrm{BA}^{2}$, Narayana Prasad MD, MPH, $\mathrm{MS}^{2}$, \\ Mayra Volquez $\mathrm{MSc}^{2,4}$ \\ ${ }^{1}$ Medical officer, Miracle Medical Centre, St. Thomas, Jamaica \\ ${ }^{2}$ Public Health literacy, www.publichealthliteracy.org USA \\ ${ }^{3}$ Walden University, USA \\ ${ }^{4}$ Colorado State University, USA
}

\section{ABSTRACT}

Aim: The COVID-19 pandemic is an unprecedented global crisis affecting several countries, including Jamaica. The risk perception of a crisis is shaped by both real hazards and perceived threats. Therefore, a cross-sectional research survey was conducted in August 2020 to evaluate Jamaicans' perceived health concerns during COVID-19.

Methods: A cross-sectional randomized online questionnaire survey was sent to a total of 268 participants. As a result, 92 people (34\%) responded to the online questionnaire survey in August 2020, and SPSS analyzed the data.

Results and Discussion: Out of 92 respondents, $78 \%$ were females, $22 \%$ were males, and more than $70 \%$ of participants were over 35 yrs. old. $52 \%$ of participants perceived the country was not prepared for a crisis such as COVID-19. The biggest concerns during this crisis were family health (39\%), economic hardship (28\%), societal health (20\%), and personal health (13\%). In personal health, the majority of Jamaicans were concerned about physical and mental health during this crisis.

Conclusion: The responding and understanding of the ongoing COVID-19 pandemic are functions of both natural threats and perceived risks. The interaction of physical health and risk perception is complex and dependent on understanding the crisis, uncertainty, and destructive potential. The results suggest a crisis burden on mental health in addition to physical health; therefore, an effective campaign should be planned to prevent a deepening mental health crisis. This research study suggests a potential burden on societal health in healthcare delivery systems and other public health services. The awareness program should be promoted to motivate and help the Jamaicans to handle the crisis. The policymakers should consider working with government, science, and faith-based institutions to develop a multidisciplinary framework to control the COVID-19 pandemic's negative impact on Jamaican society.

KEYWORDS: Mental health, COVID-19, pandemic, healthcare crisis

\section{BACKGROUND}

COVID-19 pandemic needs no introduction. COVID-19 has affected millions of people across the world, and hundreds of countries are in crisis. No other single crisis in the current living generation has affected people's lives as COVID-19. Therefore, all information, including scientific and social relevance in addition to clinical and medical understanding, is crucial to guide public health policies. There has been a massive disruption of day-to-day human lives, including churches, schools, businesses, and any social gatherings during their lifetime. Understanding people's perceptions of the risks and their reaction to the crisis such as COVID-19 is essential to design meaningful and practical solutions. ${ }^{1}$

Jamaica, one of the most popular English-speaking Caribbean countries, is a common tourist destination for decades. In 2019, over 4 million tourists visited the country, double the number of tourists compared to 2000. In 2019, Jamaica earned 3.5 Billion US dollars from tourism. Due to COVID-19 and its related restrictions, tourism dropped to one million affecting hotels and restaurants businesses with $30 \%$ decline in the revenue. According to Work Bank, this may have abolished the progress made in poverty reduction over some years. ${ }^{8}$ (Figure 1 ) 


\section{Figure 1- Actual and projected poverty rates and real GDP per capita}

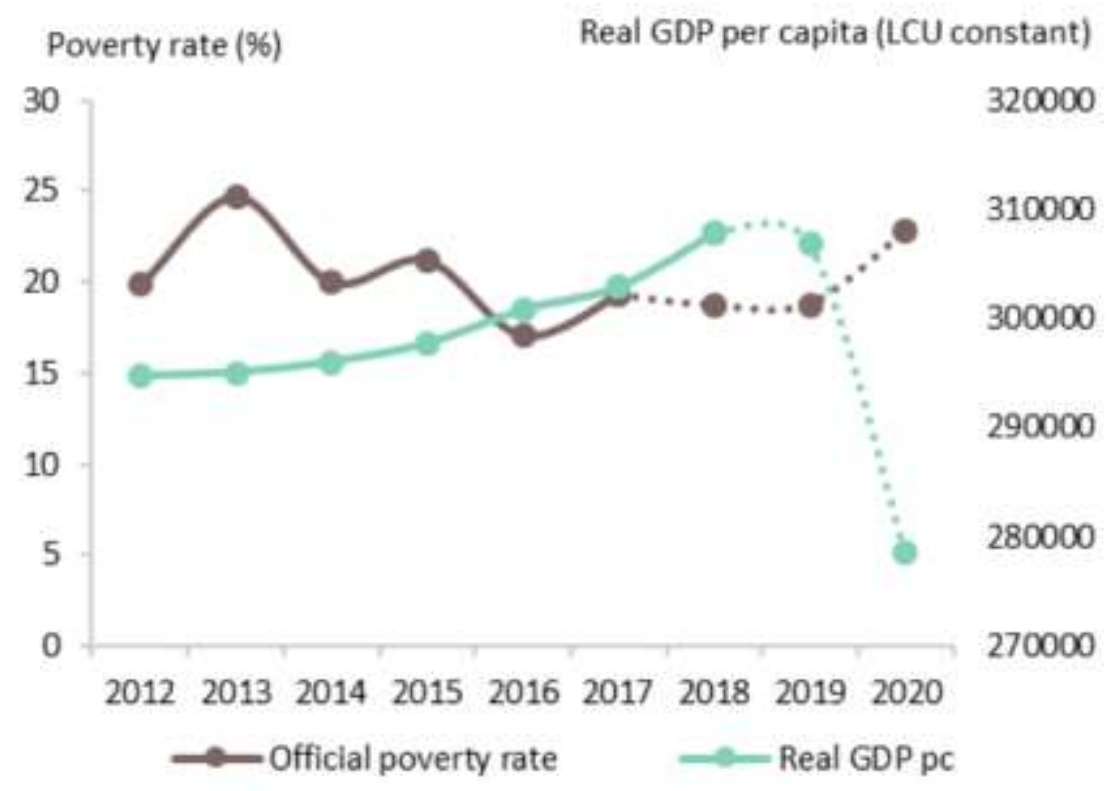

Source: Statistical Institute of Jamaica and World Bank staff calculations.

In addition to the extraordinary nature of economic hardship and peril of millions of Jamaicans livelihood, Jamaicans have endured physical and mental health sufferings. Societal and cultural norms sway the risk perception during a health crisis of any kind. The risk perception can impinge on risk management because of psychological extrapolation. ${ }^{6,7}$

Even a small cultural factor can influence the management of crises and emergencies. ${ }^{3}$ Many social and cultural norms can affect the way people perceive risk during a crisis. Public health compliance is significantly correlated with individual and hence the community risk perception. Thus, both socio-cultural context and individual knowledge shape the perceived risk. ${ }^{14}$ This relates to protection-motivation theory (PMT).

According to PMT, disease prevention and health promotion are affected directly by perceived risk and therefore affect response efficacy, self-efficacy, and supplementary adaptive behaviors ${ }^{4}$. While vigorous and efficiently working healthcare system and a successful COVID-19 vaccination campaign are crucial and essential, health messaging, communication, knowledge building, and adoption of non-pharmacological interventions are similarly and strategically important. The Jamaican ministry of health has advocated. ${ }^{5}$ Various measures during this crisis that is considered universal. These involve hand washing, wearing face masks, and keeping physical distance during any interactive dealings in public and private setup. The success of these measures is directly correlated to individual and collective understanding of the significance of the measures. ${ }^{2}$ The biological threat of the pandemic and its trajectory depends on the country's social and cultural makeup. ${ }^{15}$ To successfully implement public health protocols, assessment of accurate risk perception is critical. ${ }^{4}$ According to the psychometric paradigm and subjective-expected approach, the public view of the risk is a factor for successful public health protocol implementation. ${ }^{10}$ These risks are categorized under unknown risks and are described as new, non-observable, relatively less quantifiable, unknown to science with delayed effects. ${ }^{16}$

COVID 19 cases in Jamaica over time (Oct 2020 to Sept 2021) 10,11

\begin{tabular}{|l|l|l|l|l|l|}
\hline & Population & Total cases & Total deaths & case per $\mathbf{1 0 0 , 0 0 0}$ & $\begin{array}{l}\text { Fully vaccinated } \\
\text { percentage }\end{array}$ \\
\hline $\begin{array}{l}\text { Jamaica } \\
\text { As of Oct 8, 2020 }\end{array}$ & 2.9 million & 6408 & 101 & $\begin{array}{l}\text { None } \\
\text { (No } \begin{array}{l}\text { vaccines } \\
\text { available) }\end{array}\end{array}$ \\
\hline $\begin{array}{l}\text { Jamaica } \\
\text { As of Sept 1, 2021 }\end{array}$ & 2.9 million & 68482 & 1549 & 218 & $4.9 \%$ \\
\hline
\end{tabular}




\section{Perceived Health Concerns During COVID 19 Crisis Amongst Jamaicans}

\section{METHODS AND MATERIAL}

Between May 2020 and August 2020, we conducted a cross-sectional survey in a representative sample of the Jamaican population 15 years and over. A randomized online questionnaire survey was sent to 268 people, out of which 91 people (34\%) responded. Various social media platforms such as WhatsApp, Facebook, LinkedIn, and Public Health Literacy webinar attendees were utilized to collect the participants' responses. Thus, the participants were a representative sample of the Jamaican population. The survey was administered in a web-based browser using Survey Monkey and the average time to complete the survey was less than three minutes to complete. There was no financial incentive offered to the participants. The questionnaire was designed in English, the official and the primary language in Jamaica. The survey questions were not intrusive nor collected any information that reveal the identity of the participants. The Questions were designed to measure affective, cognitive, and temporal-spatial behaviors of the participants' towards the risk perception of COVID 19. No individual COVID 19 disease status nor other biological markers of health were collected. The data was analyzed using Statistical Package for The Social Sciences (SPSS).

\section{Categories used in the thematic analysis:}

\begin{tabular}{|l|l|}
\hline Categories used & \multicolumn{1}{|c|}{ Item question used } \\
\hline Demographic variation by age and gender & Samples stratified by age and gender \\
\hline Personal knowledge & Do you believe your country was prepared by COVID-19? \\
\hline Social amplification & $\begin{array}{l}\text { What is your biggest health concern about your personal health? } \\
\text { What is your biggest health concern about your loved ones? }\end{array}$ \\
\hline Overall Perceived risk & $\begin{array}{l}\text { What is the biggest concern during this crisis? } \\
\text { Which one of the public health issues your country is not doing well? }\end{array}$ \\
\hline Trust in the institutions & Who do you think should be leading the crisis? \\
\hline
\end{tabular}

\section{RESULTS AND DISCUSSION}

The descriptive data of the results are presented below. The gender difference in the participants was a significant finding, such that $78 \%$ were females (Chart 1). This finding correlates with other studies on risk perception on COVID 19 that women are more likely to react to public health concerns and adopt precautionary behavior than men during the COVID-19 pandemic. ${ }^{12}$ Over $75 \%$ of participants were 35 yrs. And over (Chart 1) suggesting relatively older population participation in the survey. This find ing correlates with another study on risk perception on COVID, signifying perception of risk severity increases with age. ${ }^{13} 70 \%$ of the participants had a bachelor's degree or higher educational background.

In response to the category of personal knowledge of this crisis - do you believe your country was prepared for the COVID-19 pandemic; Jamaicans were divided equally, $50 \%$ saying yes, $50 \%$ saying no. Next, in response to the category of social amplification - what is your biggest concern about your health during the crisis; Jamaicans were concerned about physical health (38\%), mental health (30\%), emotional health (16\%), and social health (14\%) (Chart 5). In response to the second measure of social amplification - what is your biggest concern about the health of your loved ones during the crisis; Jamaicans were relatively more concerned about the physical health of loved ones (48\%), followed by mental health (28\%), and emotional health (14\%) (Chart 6). In response to the category of overall risk perception - what is your biggest concern during this time; Jamaicans were concerned about family health (38\%), economic hardship (27\%), social health (20\%), least concerned about personal health (13\%) (Chart 7). Finally, in response to the second measure of overall risk perception - which one of the public health issues your country is not doing well; more than $50 \%$ of Jamaicans responded to multiple public health issues such as domestic and physical abuse, sexual abuse, child abuse, mental health concerns, and economic hardship.

In response to the category of trust in the institutions, $81 \%$ of participants responded that all institutions, including science, public policy, and faith-based institutions, should lead the COVID-19 crisis as a team (Chart 8). This aligns with socio-cultural norms of the communities that respect and supports the framework of collective efforts in resolving crises such as infectious disease outbreaks. $^{12}$ 
Perceived Health Concerns During COVID 19 Crisis Amongst Jamaicans

Chart 1:

Gender

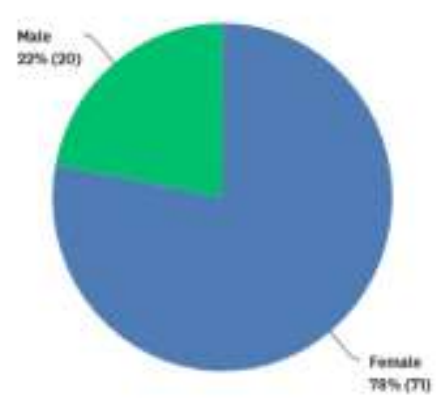

Age

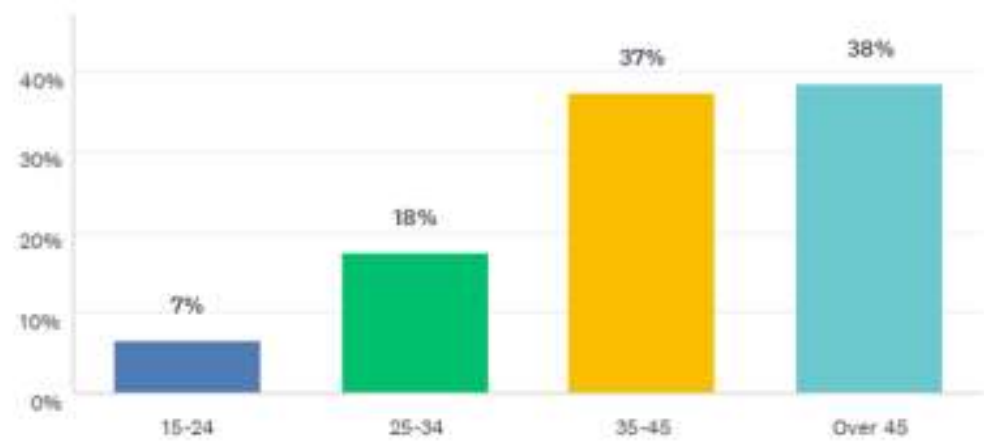

Chart 2:

What is your highest educational background?

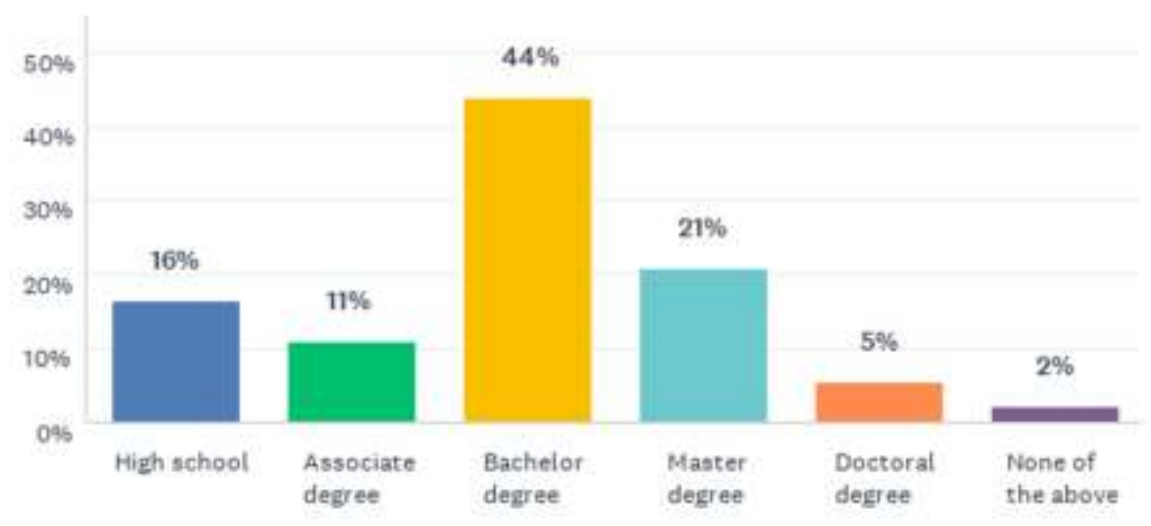

Chart 3:

What is your biggest concern during this time?

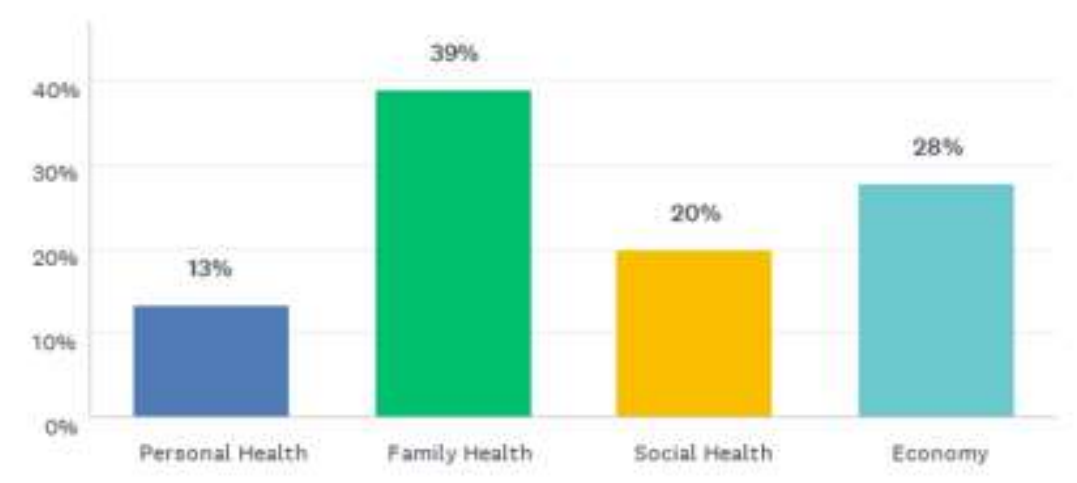

Chart 4:

Do you believe your country was prepared for a crisis such as COVID-19?

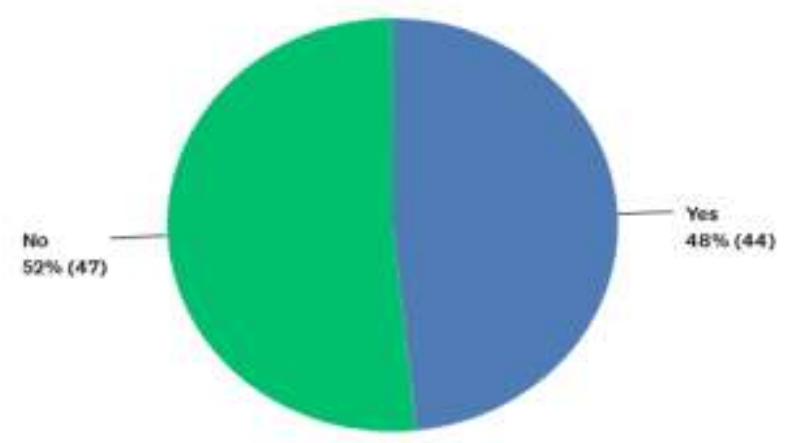


Perceived Health Concerns During COVID 19 Crisis Amongst Jamaicans

Chart 5:

What is your biggest concern about your personal health during this crisis?

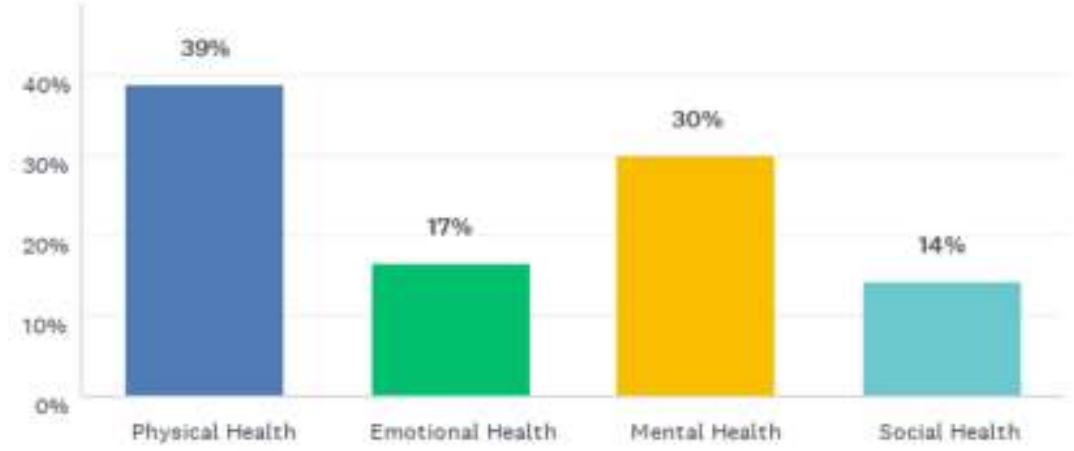

Chart 6:

What is your biggest concern about the health of your loved ones?

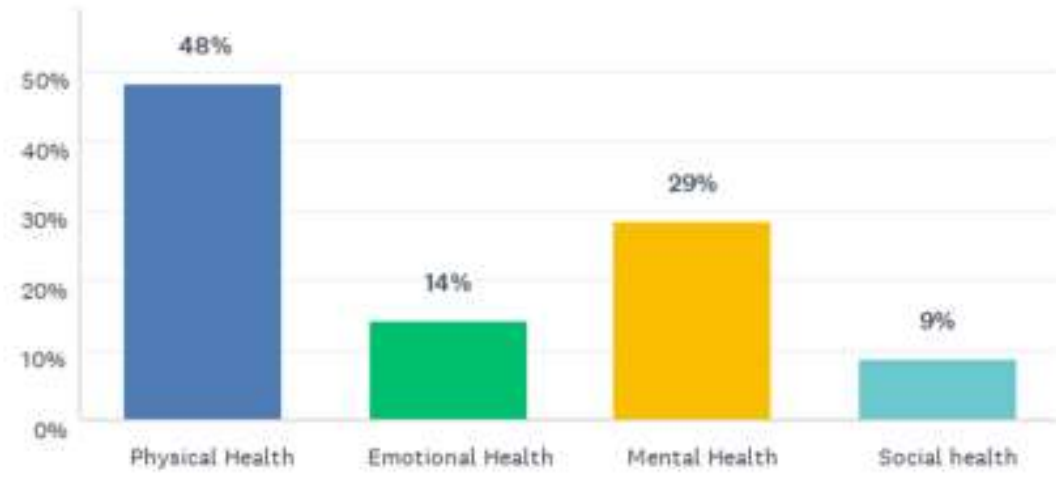

Chart 7:

What is your biggest concern during this time?

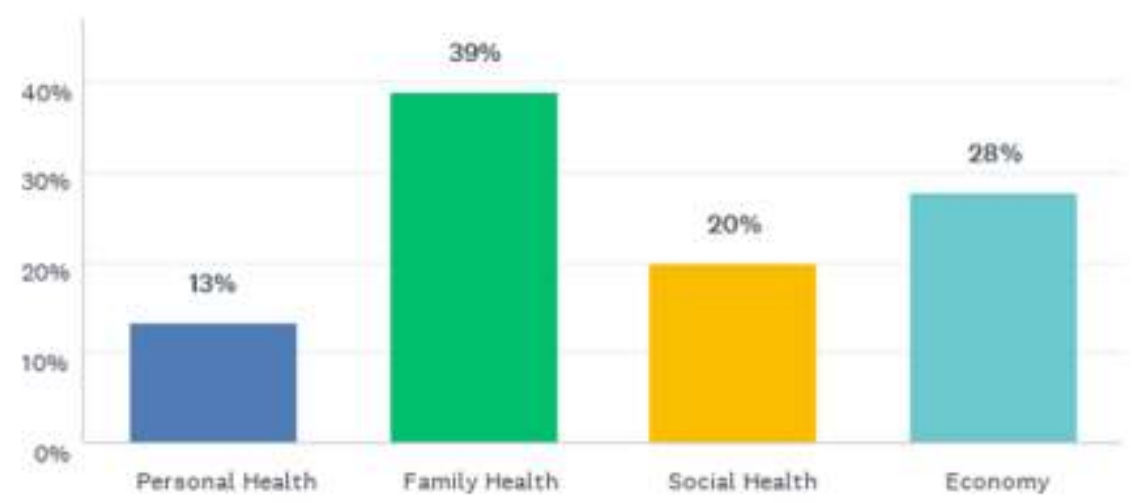

Chart 8:

Which one of the Public Health Issues your country is not doing well?

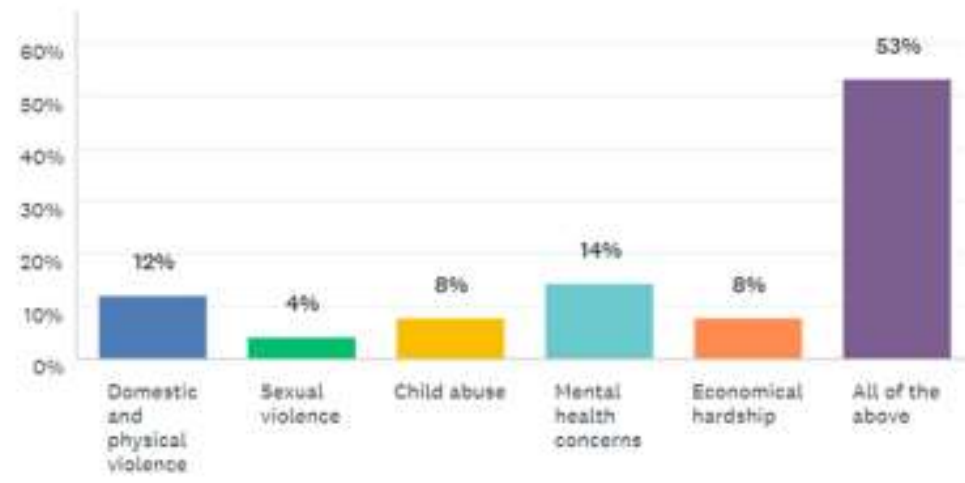




\section{Perceived Health Concerns During COVID 19 Crisis Amongst Jamaicans}

Chart 9:

\section{Who do you think should be leading in a crisis such as COVID-19?}

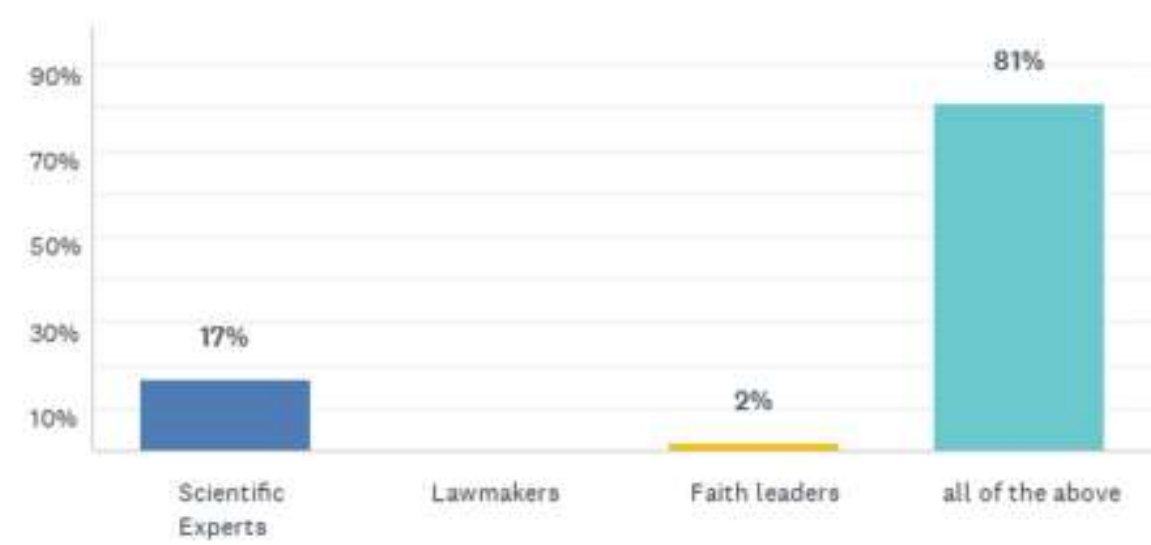

There were several limitations to this study. Since this is a cross-sectional survey, this is a snapshot of the sampled population at one particular point in time, hence does not assess change in risk perception and behavior change in the same people over time. The second limitation is that this is a descriptive insight in the risk perception, hence no correlational nor causal effects can be determined. The third limitation is the lack of a larger sample of Jamaicans, particularly the vulnerable segment of the population.

\section{CONCLUSION}

The responding and understanding of the ongoing COVID-19 pandemic are functions of both natural threats and perceived risks. The interaction of physical health and risk perception is complex and dependent on understanding the crisis, uncertainty, and destructive potential. The results suggest a crisis burden on mental health in addition to physical health; therefore, an effective campaign should be planned to prevent a deepening mental health crisis. This research study suggests a potential burden on societal health in healthcare delivery systems and other public health services. The awareness program should be promoted to motivate and help the Jamaicans to handle the crisis. The policymakers should consider working with government, science, and faith-based institutions to develop a multidisciplinary framework to control the COVID-19 pandemic's negative impact on Jamaican society.

\section{REFERENCES}

1) Science in the time of COVID-19. (2020, April 8). Nature Structural \& Molecular Biology. https://doi.org/10.1038/s41594-020-0423-7.

2) Dryhurst, S., Schneider, C. R., Kerr, J., Freeman, A. L. J., Recchia, G., van der Bles, A. M., Spiegelhalter, D., \& van der Linden, S. (2020). Risk perceptions of COVID-19 around the world. Journal of Risk Research, 23(7-8), 994-1006. https://doi.org/10.1080/13669877.2020.1758193

3) Bergeron W. P. (2015). Considering culture in evacuation planning and consequence management. Journal of emergency management (Weston, Mass.), 13(2), 87-92. https://doi.org/10.5055/jem.2015.0222

4) FLOYD, D. L., PRENTICE-DUNN, S., \& ROGERS, R. W. (2000). A Meta-Analysis of Research on Protection Motivation Theory. Journal of Applied Social Psychology, 30(2), 407-429. https://doi.org/10.1111/j.1559-1816.2000.tb02323.x

5) Horton, R. (2020). Offline: The lessons of smallpox eradication for COVID-19. The Lancet, 396(10267), 1951. https://doi.org/10.1016/s0140-6736(20)32713-6

6) Slovic P. (1987). Perception of risk. Science (New York, N.Y.), 236(4799), 280-285. https://doi.org/10.1126/science.3563507

7) 'Zizza, A., Guido, M., Recchia, V., Grima, P., Banchelli, F., \& Tinelli, A. (2021). Knowledge, Information Needs and Risk Perception about HIV and Sexually Transmitted Diseases after an Education Intervention on Italian High School and University Students. International journal of environmental research and public health, 18(4), 2069. https://doi.org/10.3390/ijerph18042069

8) Jamaica Earned US\$3.64 Billion From Tourism In 2019, Welcomed 4.3 Million Visitors - Jamaica Information Service. (n.d.). Jamaica Information Service - The Voice of Jamaica. Retrieved September 5, 2021, from https://jis.gov.jm/jamaica-earned-us3-64-billion-from- tourism-in-2019-welcomed-4-3-million-visitors/

9) Prasad, N., Cruz, M., Prasad, S., \& Denny, H.M. (2021): Health risk perception of COVID-19 in Caribbean countries, Ann Trop Med \& Public Health; 22(S01): SP241103. DOI: http://doi.org/10.36295/ASRO.2021.241103 


\section{Perceived Health Concerns During COVID 19 Crisis Amongst Jamaicans}

10) Times, T. N. Y. (2021, September 5). Coronavirus World Map: Tracking the Global Outbreak. The New York Times. https://www.nytimes.com/interactive/2021/world/covid-cases.html

11) Alqahtani, M. M., Arnout, B. A., Fadhel, F. H., \& Sufyan, N. S. S. (2021). Risk perceptions of COVID-19 and its impact on precautionary behavior: A qualitative study. Patient Education and Counseling. Published. https://doi.org/10.1016/j.pec.2021.02.025

12) Rosi, A., van Vugt, F. T., Lecce, S., Ceccato, I., Vallarino, M., Rapisarda, F., Vecchi, T., \& Cavallini, E. (2021). Risk Perception in a Real-World Situation (COVID-19): How It Changes From 18 to 87 Years Old. Frontiers in Psychology, 12. https://doi.org/10.3389/fpsyg.2021.646558

13) Gerrard, M., Gibbons, F. X., \& Bushman, B. J. (1996). Relation between perceived vulnerability to HIV and precautionary sexual behavior. Psychological Bulletin, 119(3), 390-409. https://doi.org/10.1037/0033-2909.119.3.390

14) Malecki, K. M. C., Keating, J. A., \& Safdar, N. (2020). Crisis Communication and Public Perception of COVID-19 Risk in the Era of Social Media. Clinical Infectious Diseases. 1-6. https://doi.org/10.1093/cid/ciaa758

15) Leppin, A., \& Aro, A. R. (2009). Risk Perceptions Related to SARS and Avian Influenza: Theoretical Foundations of Current Empirical Research. International Journal of Behavioral Medicine, 16(1), 7-29. https://doi.org/10.1007/s12529-0089002-8 